\title{
Médiévales
}

Langues, Textes, Histoire

58 | printemps 2010

Humanisme et découvertes géographiques

\section{Pierre BAUDUIN, Le Monde franc et les Vikings (VIII ${ }^{e}-\mathrm{X}^{e}$ siècle), Paris, Albin Michel, 2009, 455 p.}

\section{Rodolphe Keller}

\section{(2) OpenEdition}

\section{Journals}

Édition électronique

URL : https://journals.openedition.org/medievales/6037

DOI : $10.4000 /$ medievales.6037

ISSN : 1777-5892

\section{Éditeur}

Presses universitaires de Vincennes

\section{Édition imprimée}

Date de publication : 30 juin 2010

Pagination : $172-175$

ISBN : 978-2-84292-260-3

ISSN : 0751-2708

\section{Référence électronique}

Rodolphe Keller, «Pierre Bauduin, Le Monde franc et les Vikings (VIII-xe siècle), Paris, Albin Michel, 2009, 455 p. », Médiévales [En ligne], 58 I printemps 2010, mis en ligne le 17 janvier 2011, consulté le 24 avril 2022. URL : http://journals.openedition.org/medievales/6037 ; DOI : https://doi.org/10.4000/ medievales. 6037

Ce document a été généré automatiquement le 24 avril 2022.

Tous droits réservés 


\title{
Pierre BAUDUIN, Le Monde franc et les Vikings (VIII ${ }^{e} X^{e}$ siècle), Paris, Albin Michel, 2009, 455 p.
}

\author{
Rodolphe Keller
}

1 L'ouvrage de Pierre Bauduin, consacré aux relations entre le monde franc et les sociétés scandinaves du $\mathrm{VIII}^{\mathrm{e}}$ au x $\mathrm{x}^{\mathrm{e}}$ siècle, renouvelle en profondeur l'approche de cette question. L'auteur part du constat que, si les sources insistent beaucoup sur les violences des Vikings présents dans le monde franc, leurs incursions donnent lieu à des relations d'échanges complexes. Il entend donc examiner "les modes de pacification et de médiation entre les Scandinaves et les Francs » (p. 11), dans la continuité de la recherche historique la plus récente. Les historiens ont tout d'abord vu dans la manière dont les pouvoirs francs ont composé avec les Vikings une désastreuse capitulation. Mais, depuis une vingtaine d'années, la recherche a développé une approche plus fine de l'impact réel de la présence scandinave sur le monde franc, des caractères de leur installation, de l'attitude des pouvoirs francs. L'auteur aborde ainsi la question sur la base de la notion d'" accomodation », qui se rapporte au " processus [...] de régulation des relations entre nouveaux venus et communautés établies ou aux stratégies de coexistence entre ces groupes " (p. 36), afin de mettre en lumière les "mécanismes qui ont pu jouer, ou non, en faveur de l'intégration des Vikings dans les sociétés autochtones » (p. 25).

2 Une première série de chapitres traite des cadres généraux de la négociation (ch. 2-3). Il n'existe pas de traité conservé concernant les relations entre les Francs et les Scandinaves, mais l'analyse de la terminologie montre l'existence de plusieurs formes d'accords, comme la pax, ou l'établissement de relations d'amicitia. De nombreuses transactions ne sont pas évoquées en tant que telles dans les sources, mais peuvent parfois en être déduites. Les nombreux miracles concernant les Normands sont particulièrement intéressants à cet égard. Au début des années 870, saint Malo sauve les villageois de Cherrueix des Normands, après qu'ils lui ont fait don de quatre deniers, ce qui cacherait un tribut versé aux assaillants. Les récits des contemporains sont 
souvent biaisés par des facteurs idéologiques. L'évocation des destructions normandes peut cacher une critique du pouvoir royal, incapable de protéger efficacement le territoire, alors que le récit d'une paix permet de glorifier le rex pacificus. Dans ce contexte, la "grammaire de base de ces relations passe par les éléments les plus universellement reconnus dans les sociétés anciennes: les serments, les otages, l'échange de présents» (p. 86), bien qu'il existe certaines spécificités. Les lieux de négociations sont soigneusement préparés. La conclusion de la paix intervient souvent aux frontières, car cela permet de mettre les partenaires sur un pied d'égalité, comme lors des règlements frontaliers de 811 entre Francs et Danois. Les assemblées du royaume, au cours desquelles sont reçues les ambassades étrangères, constituent un autre cadre privilégié de la négociation. De nombreux rituels interviennent lors de ces rencontres, comme des banquets ou des échanges de dons, qui peuvent parfois masquer des prélèvements tributaires.

Dans une seconde série de chapitres, l'auteur aborde quelques dossiers particuliers, choisis pour leur richesse documentaire (ch. 4 -7). Pour chacun d'entre eux, il mène une analyse textuelle dont il faut souligner la finesse et la richesse. Un premier dossier concerne le baptême de Harald Klak en 826, qui illustre le modèle d'intégration proposé par le monde franc aux élites du monde scandinave. Le récit d'Ermold le Noir montre une véritable "transformation des hôtes de Louis en une communauté d'hommes partageant avec les Francs les éléments d'une identité commune. »(p. 135). Le baptême de Harald, de sa famille et de sa suite marque leur entrée dans la communauté du populus christianorum. Le parrainage de Louis le Pieux, inspiré de pratiques byzantines, permet à Harald d'entrer dans la « famille des rois » (A. Angenendt), par le biais de la parenté spirituelle. L'intégration passe également par une série de rituels profanes, comme le banquet et la chasse, constitutifs de la sociabilité aristocratique, qui représentent autant d'étapes de l'entrée de Harald dans la société franque. Le dossier suivant traite des événements de 845 , lorsque le monde franc subit une importante série d'attaques. Le chef danois Ragnar envahit la région de la Seine et obtient le paiement d'un tribut par Charles le Chauve. Ensuite, il est probablement à l'origine de l'attaque de Hambourg, avant de retourner au Danemark. D'après les sources, il est alors atteint d'une mort miraculeuse, qui effraie le roi Horich, ce qui le pousse à rendre les trésors et les captifs chrétiens. En fait, ce dernier a sans doute dû se désolidariser de ses compatriotes dans le contexte des négociations avec Louis le Germanique. Les récits sur le paiement $\mathrm{du}$ tribut par Charles le Chauve illustrent les enjeux historiographiques. L'auteur de la Translatio Sancti Germani, qui fournit des détails importants, ne l'évoque pas, contrairement à Aimon d'Auxerre, lorsqu'il reprend le texte dans ses Miracula sancti Germani, afin d'illustrer a posteriori le succès de la politique de Charles le Chauve. Lors des événements de 873, les enjeux sont tout aussi complexes. Les rois danois concluent un accord sur une question frontalière avec Louis le Germanique, avant de modifier leur attitude et d'adopter une posture de franche soumission. L'évolution des relations se comprend mieux si l'on tient compte du rapprochement entre Louis le Germanique et Roric, un Danois installé en Frise, ce qui semble inquiéter les rois danois et les amener à se montrer plus conciliants. Cette même année, Charles le Chauve conduit un siège contre les Normands installés à Angers, ce qui donne lieu à des récits divergents. Hincmar présente la résolution du siège d'Angers comme un succès royal, mais une tradition historiographique, dont témoigne par exemple la chronique de Réginon de Prüm, se révèle nettement plus réservée. Un dernier dossier porte sur les événements de 882. Le siège des Vikings 
installés à Asselt par Charles le Gros est connu par de nombreux récits. L'auteur de la version mayençaise des Annales de Fulda est résolument hostile à Charles le Gros et en donne une image très critique. Il présente un Charles le Gros qui capitule devant les païens et leur paie un tribut, au lieu de combattre les ennemis du peuple chrétien. L'auteur de la version bavaroise, au contraire, mène un effort pour « transformer la défaite en victoire " (p. 215). En fait, l'accord de 882 constitue surtout un exemple d'intégration d'un chef viking au monde franc. Godfrid est baptisé, sur le modèle de Harald Klak, reçoit un territoire en Frise et épouse Gisla, la fille de Lothaire II, ce qui lui permet d'intégrer les réseaux politiques francs.

Une troisième série de chapitres porte sur l'analyse du " contexte et [de] la sociologie de la médiation" (p. 225). Après la conquête de la Saxe par Charlemagne et l'établissement de relations suivies avec les Danois, un groupe de Grands se démarque, spécialisé dans les affaires nordiques (ch. 8). Ces personnages exercent une influence considérable en Saxe et leur rôle de médiateurs pourrait s'expliquer par l'existence de relations étroites entre les élites saxonnes et danoises. Au cours du IX siècle, ils continuent à jouer un rôle important. Le partage de l'empire profite alors à Louis le Germanique, qui a les moyens de monopoliser les relations avec les Danois. Un autre groupe de médiateurs est composé par les acteurs de la mission d'évangélisation des Scandinaves (ch. 9). Ansgar, évêque de Hambourg, "apôtre du Nord ", dispose de réseaux dans le royaume danois, mais aussi en Suède, et sert d'intermédiaire. Il entretient des relations d'amicitia avec le souverain danois Horich $\mathrm{I}^{\mathrm{er}}$, et devient même son proche conseiller. Avec l'accroissement de la pression viking vers le milieu du $\mathrm{IX}^{\mathrm{e}}$ siècle, l'analyse se porte vers les formes de la médiation qui interviennent dans le cadre des incursions scandinaves (ch. 10). Les Grands jouent un rôle toujours croissant. Le traité de Saint-Clair-sur-Epte en 911 apparaît comme un compromis entre Charles le Simple, Rollon, Robert de Neustrie et Herbert de Vermandois. Par ailleurs, l'intégration des Normands devient un enjeu de la compétition entre les élites, dans la mesure où celle-ci s'ouvre à de nouveaux venus. Le chef Weland est rétribué par Charles le Chauve pour chasser d'autres Vikings de leur repaire d'Oissel. Des Normands comme Roric obtiennent des terres en Frise et adoptent le mode de vie franc. Godfrid intègre le réseau aristocratique franc en 882, par son mariage avec Gisla, mais est tué en 885 lors d'un complot organisé par Henri de Babenberg, ce qui témoigne probablement des tensions au sein de l'aristocratie face à l'introduction de nouveaux partenaires dans les circuits de l'échange compétitif. Les évêques francs jouent également un rôle dans les négociations avec les Vikings (ch. 11). Cependant, leurs interventions demeurent discrètes, mais cela reflète peut-être les réticences des auteurs à les illustrer dans un rôle de médiateurs avec des païens. Il est étonnant de constater qu'ils semblent rétifs à s'impliquer dans la conversion des païens présents, ce qui contraste avec l'effort missionnaire mené en direction du monde scandinave dans les années 820-860. Cette attitude change au début du $\mathrm{x}^{\mathrm{e}}$ siècle, lorsqu'il n'est plus possible de s'opposer au principe de l'entente entre Francs et Vikings. Un dernier chapitre concerne les Francs ayant noué des relations avec des Normands contre d'autres Francs (ch. 12). Les cas sont rares et peu probants. Certaines sources suggèrent la collusion de Lothaire avec les Normands, durant les années 830, mais ces soupçons s'expliquent essentiellement par la propagande contre Lothaire. De la même manière, la responsabilité du comte Lambert II dans l'attaque viking contre Nantes en 843, évoquée par certains récits, semble surtout s'expliquer par une mauvaise image du personnage, en raison de ses relations avec les Bretons. En revanche, l'alliance de Pépin 
II d'Aquitaine avec les Normands, dans le cadre de sa révolte contre Charles le Chauve, est avérée. Dans cette affaire comme dans d'autres, le clergé craint que de telles alliances représentent une menace pour la foi de ceux qui s'y prêtent, comme l'illustre une lettre de Foulques de Reims à Charles le Simple, lors de son alliance avec le Viking Hundeus, en 897.

5 Pierre Bauduin peut ainsi conclure en soulignant les enjeux de l'analyse textuelle, qui doit dépasser l'idée simpliste d'un " manichéisme réel ou supposé des sources » et d'un parti clérical des auteurs, afin de tenir compte de motivations plus complexes et des " clivages qui tiennent bien davantage aux représentations du pouvoir, aux stratégies de communication publique, aux rivalités de la société politique du temps. » (p. 343.) L'analyse des sources illustre la complexité des enjeux qui caractérisent l'interaction entre Francs et Normands, comme l'attitude du pouvoir royal face aux stratégies de médiation, la diversité des acteurs et de leurs intérêts, l'importance croissante des élites, ou les facteurs de coexistence. Le monde franc dispose d'un modèle d'intégration, mais celui-ci "fonctionne moins dans le sens d'une adaptation réciproque que comme l'acceptation unilatérale des cadres francs. » (p. 347.) Enfin, la naissance de la principauté de Rouen, au début du xe siècle, " s'inscrit dans la logique de l'accomodation analysée dans ce livre. » (p. 347). Son succès tient à la capacité des ducs normands à mieux s'intégrer dans le réseau des élites princières du royaume que ne l'avaient fait d'autres chefs vikings.

6 Cette étude est donc construite sur la base d'une approche précise et l'on cherchera en vain un tableau exhaustif des relations franco-scandinaves. L'auteur dégage les formes complexes de la coexistence, sur la base des développements les plus récents de la recherche médiévale. Il s'inscrit dans la continuité d'une historiographie qui, en dépassant une vision simpliste de l'opposition entre Francs et Vikings, a voulu dédramatiser l'intensité du choc, en insistant sur les formes d'échanges, au risque, peut-être, de parfois sous-estimer le caractère violent de ces confrontations, qui soulèvent également nombre de questions, susceptibles d'être reprises dans une optique renouvelée. Il demeure que cette étude, riche et remarquable, représente un apport fondamental dans la recherche sur la question. L'ouvrage de Pierre Bauduin apporte également un matériel extrêmement utile pour tout chercheur qui s'intéresse à cette documentation, tant du point de vue de l'analyse des sources et des discussions critiques sur les dossiers constitués, que du point de vue de la bibliographie qui montre le renouvellement de ces questions dans la recherche internationale. 